Pacific Journal of Mathematic 


\title{
SEMI-SIMPLE CLASSES IN CHEVALLEY TYPE GROUPS
}

\author{
N. Burgoyne and C. Williamson
}

A practical method is given for finding the classes and centralizers for arbitrary $p^{\prime}$-elements in the automorphism group of a Chevalley type group over a field of characteristic $p$.

1. Introduction. In the study of finite simple groups it is important to know their conjugacy classes and the structure of the corresponding centralizer subgroups. For the alternating groups the results are well known; for the sporadic groups the calculations are special to each group. In this article the authors will study the semisimple classes in Chevalley type groups. Different methods are required for their unipotent classes.

Our approach is to work, as far as possible, in the algebraic group $G$ corresponding to the given finite Chevalley type group. If $t$ is a semi-simple element in $G$ then, in general, $C_{G}(t)$ is not connected and its component of the identity is not semi-simple but only reductive. Since certain applications [3] require the structure of centralizers of pairs of commuting semi-simple elements we are led to study the rather general situation described in $\S 2,3$, and 4 . The underlying theory for these sections is quite simple and is based on essentially two results; (i) the algorithm which leads to the fundamental domain $\mathscr{F}_{d}$ of 2.4 , for this see [2], [14] or Appendix 2, and (ii) a general result about algebraic groups, see $[14, \S 7]$, which allows one to reduce questions about semi-simple elements in $G$ to linear algebra problems in certain lattices. In a given case, once the situation in $G$ is clear, the step down to the finite group is easily done by application of Lang's theorem, see [11], [13] and $\S 5$ below.

In two unpublished notes [4], [5] this approach was used to calculate (i) the classes of involutions at odd characteristic and (ii) the 3-elements at even characteristic in $\operatorname{Aut}(L)$ for all finite Chevalley type groups $L$. We also described the layer of $C_{L}(t)$. Rather than reproduce these results, we include, in Appendix 1, the structure of certain centralizer subgroups that are of interest for current work on simple groups of component type, see for example [7], [9].

We are indebted to the fundamental paper of Steinberg [14] for the basic theory. Earlier work on these question occurs in Abe [1], Ree [12] and Iwahori [10]. In fact the starting point for our work was the attempt to put the ideas of [10] in a form which would give rapid and explicit answers to the sort of questions which arise from finite group theory.

Our notation for finite groups follows [8] and for algebraic groups [6] 
and [14]. In particular, if $C$ is an algebraic group then $C^{0}$ denotes its connected component of the identity. All homomorphisms in $\$ 2$ are algebraic.

\section{Reductive groups.}

2.1. Some standard notation. $G$ is a connected reductive algebraic group over the algebraically closed field $k$. T is a maximal torus of G. Put $N=N_{G}(T)$ and $W=N / T$. If $n \in N$ let $w(n)$ denote its image in $W$.

Let $X=X(T)=\operatorname{Hom}\left(T, k^{*}\right)$ and $\Gamma=\Gamma(T)=\operatorname{Hom}\left(k^{*}, T\right)$, where $k^{*}$ is the multiplicative group of $k$. They are $Z$-lattices of rank $r=$ dimension of $T$. Identify $\Gamma$ with the $Z$-dual of $X$ by putting $\chi(\eta(\zeta))=\zeta^{\eta(x)}$, where $\chi \in X, \eta \in \Gamma, \zeta \in k^{*}$.

The root system of $G$ relative to $T$ is denoted by $\Sigma$ and consists of all $\alpha \in X$ for which there exists an isomorphism $x_{\alpha}$ of the additive group $k_{+}$ into $G$ satisfying $t x_{\alpha}(\zeta) t^{-1}=x_{\alpha}(\zeta \alpha(t))$ for $t \in T, \zeta \in k$.

If $\alpha \in \Sigma$ the subgroup $U_{\alpha}=x_{\alpha}\left(k_{+}\right)$is uniquely determined. Let $T_{\alpha}=T \cap\left\langle U_{\alpha}, U_{-\alpha}\right\rangle$, a one-dimensional torus, and define the co-root $\hat{\alpha} \in \Gamma$ by $\hat{\alpha}\left(k^{*}\right)=T_{\alpha}$ and $\hat{\alpha}(\alpha)=2$. If $N_{\alpha}=N \cap\left\langle U_{\alpha}, U_{-\alpha}\right\rangle$ there exists $n_{\alpha} \in N$ such that $N_{\alpha}=\left\langle T_{\alpha}, n_{\alpha}\right\rangle$. Let $w_{\alpha}=w\left(n_{\alpha}\right)$ then $W=\left\langle w_{\alpha}: \alpha \in \Sigma\right\rangle$ and $w_{\alpha}$ permutes $\Sigma$ as follows: $w_{\alpha} \beta=\beta-\hat{\alpha}(\beta) \alpha$.

If $B$ is a Borel subgroup of $G$ which contains $T$ let $\Pi$ denote the resulting set of simple roots in $\Sigma$ and $\Sigma^{+}$the corresponding positive roots.

Let $E=E(G)$ be the unique maximal connected semi-simple subgroup of $G$ and $F=F(G)$ the unique torus in $G$ satisfying $E F \equiv G$, $[E, F]=1$. Note that $F \subseteq T$.

2.2. Lattices and tori. Let $X^{a d}$ be the sub-lattice of $X$ spanned by all $\alpha \in \Sigma$. Let $X^{e}$ be the unique smallest sub-lattice containing $X^{a d}$ and such that $X / X^{e}$ is torsion free. Put $X^{f}=\{\chi \in X: \hat{\alpha}(\chi)=0$ all $\alpha \in \Sigma\}$; then $X / X^{f}$ is torsion free, $X^{e} \cap X^{f}=0$ and $X / X^{e}+X^{f}$ is finite.

Let $\Gamma^{s c}$ be the sub-lattice of $\Gamma$ spanned by all $\hat{\alpha}$ where $\alpha \in \Sigma$. Let $\Gamma^{e}$ be the smallest sub-lattice containing $\Gamma^{s c}$ with $\Gamma / \Gamma^{e}$ torsion free. Put $\Gamma^{f}=\{\eta \in \Gamma: \eta(\alpha)=0$ all $\alpha \in \Sigma\}$. The pairs $X^{e}, \Gamma^{f}$ and $X^{f}, \Gamma^{e}$ are orthogonal complements, i.e. $X^{e}=\left\{\chi \in X: \eta(\chi)=0\right.$ all $\left.\eta \in \Gamma^{f}\right\}$, etc. This gives a useful way to compute $X^{e}$.

If $Y$ is any sub-lattice of $X$ define Ann $Y=\{t \in T: \chi(t)=1$ all $\chi \in Y\}$. Then Ann $Y$ is always a closed subgroup of $T$ and is a torus if $X / Y$ is torsion free. For example, $T \cap E=\operatorname{Ann} X^{f}$ and $F=$ Ann $X^{e}$. Hence, we have the natural isomorphisms, $X(T \cap E) \simeq X / X^{f}$, $\Gamma(T \cap E) \simeq \Gamma^{e}$ and $X(T / F) \simeq X^{e}, \Gamma(T / F) \simeq \Gamma / \Gamma^{f}$.

Note that $E \cap F=1$ if and only if $X^{e}+X^{f}=X$. 
2.3. Morphisms. If $f$ is a morphism of $G$ onto itself which stabilizizes $T$ then $f$ induces endomorphisms $f_{*}$ on $X$ and $f$ on $\Gamma$ as follows: if $\chi \in X, \eta \in \Gamma, t \in T, \zeta \in k^{*}$ then $f_{*} \chi(t)=\chi(f t)$ and $f \eta(\zeta)=$ $f(\eta(\zeta))$. Thus $\left(f f^{\prime}\right)_{*}=f_{*}^{\prime} f_{*}$ and $f \eta(\chi)=\eta\left(f_{*} \chi\right)$.

An example is $f=i_{n}$ where $n \in N$ and $i_{n} g=n g n^{-1}$ for $g \in G$. If $w=w(n)$ we put $i_{n} \eta=w \eta$ for $\eta \in \Gamma$. This gives a representation of $W$ on $\Gamma$. If $\alpha \in \Sigma$ one has $w_{\alpha} \eta=\eta-\eta(\alpha) \hat{\alpha}$. We adopt a common convention by letting $w$ also denote the action of $W$ on $X$ induced by the permutation of $\Sigma$ given in 2.1, together with trivial action on $X^{f}$; thus $w_{\alpha} \chi=\chi-\hat{\alpha}(\chi) \alpha$ for $\chi \in X$. Then $\left(i_{n}\right)_{*}=w^{-1}$ and so $w \eta(w \chi)=\eta(\chi)$.

2.4. Action of $W$ on $\Gamma$. Let $d$ be a fixed, positive integer and put $d \Gamma=\{d \eta: \eta \in \Gamma\}$. Consider the group $\mathscr{A}_{d}=\mathscr{A}_{d}(W, \Gamma)=$ $\{a(w, \lambda): w \in W, \lambda \in d \Gamma\}$ of all affine motions $a(w, \lambda) \mu=w \mu+\lambda$ where $\mu \in \Gamma$. We wish to describe the orbits of $\mathscr{A}_{d}$ on $\Gamma$.

Let $\mathscr{A}_{d}^{s c}=\mathscr{A}_{d}\left(W, \Gamma^{s c}\right)$ and note that $\mathscr{A}_{d}^{s c} \Vdash \mathscr{A}_{d}$. A standard result, [2], $[14, \S 1]$, or Appendix 2, states that a fundamental domain for $\mathscr{A}_{d}^{s c}$ on $\Gamma$ is given by the set

$$
\mathscr{F}_{d}=\mathscr{F}_{d}(\Gamma)=\left\{\mu \in \Gamma: 0 \leqq \mu(\alpha) \leqq d \text { all } \alpha \in \Sigma^{+}\right\}
$$

Using this result we next describe a fundamental domain for $\mathscr{A}_{d}$.

Let $\Pi^{*}$ denote the extended system of simple roots obtained by adjoining to $\Pi$ all lowest roots in $\Sigma$; one such root for each connected piece of the Dynkin diagram. Let $V$ denote the stabilizer in $W$ of $\Pi^{*}$; its elements are completely characterized by their action on the subset $\Pi^{*}$ - $\Pi$. Put, for $d=1, \mathscr{F}=\mathscr{F}_{1}$ and to each $\theta \in \mathscr{F}$ associate a unique $v_{\theta} \in V$ by: $v_{\theta} \alpha_{*}=\alpha_{*}$ if $\theta\left(\alpha_{*}\right)=0$ for $\alpha_{*} \in \Pi^{*}-\Pi$, otherwise $v_{\theta} \alpha_{*}=\alpha$ where $\alpha$ is the unique element in the orbit of $\alpha_{*}$ under $V$ which satisfies $\theta(\alpha)=1$.

$\mathscr{F}$ is a set of coset representatives for $\Gamma^{s c}$ in $\Gamma$. If $\eta \in \Gamma$ let $\theta_{\eta} \in \mathscr{F}$ satisfy $\theta_{\eta}-\eta \in \Gamma^{s c}$. We define a map $\delta_{d}$ of $\Gamma$ into $\mathscr{A}_{d}$ by $\delta_{d} \eta=a\left(v_{\theta}, d \theta\right)$ where $\theta=\theta_{\eta}$. Since $W$ centralizes $\Gamma / \Gamma^{s c}$ we see that $\delta_{d}$ is a homomorphism with kernel $\Gamma^{s c}\left(\right.$ e.g. $\left.\delta_{d}\left(\eta+\eta^{\prime}\right)=\delta_{d}(\eta) \delta_{d}\left(\eta^{\prime}\right)\right)$. It is easy to verify that the group $\mathscr{C}_{d}=\mathscr{C}_{d}(\Gamma)=\delta_{d} \Gamma$ stabilizes $\mathscr{F}_{d}$ and is a complement to $\mathscr{A}_{d}^{s c}$ in $\mathscr{A}_{d}$. Thus,

Proposition A. Any fundamental domain for $\mathscr{C}_{d}$ on $\mathscr{F}_{d}$ is also a fundamental domain for $\mathscr{A}_{d}$ on $\Gamma$.

Now consider a fixed $\mu \in \mathscr{F}_{d}$ and define the subgroups $W(\mu)=$ $\{w \in W:(1-w) \mu \in d \Gamma\}, \quad W(\mu)^{s c}=\left\{w \in W:(1-w) \mu \in d \Gamma^{s c}\right\} \quad$ and $V(\mu)=\left\{v_{\theta} \in V:\left(1-v_{\theta}\right) \mu=d \theta\right\}$. Using Proposition A and the connection between $\mathscr{F}$ and $\Gamma$ and, for (ii), a result in $[14, \S 1]$, we have 
Proposition B. (i) $W(\mu)^{s c} \leqslant W(\mu)$ and $V(\mu)$ is a complement.

(ii) $W(\mu)^{s c}=\left\langle w_{\alpha}: \alpha \in \Sigma, \mu(\alpha) \in d Z\right\rangle$.

Define the homomorphism $\bar{\delta}$ of $\Gamma$ into $V$ by $\bar{\delta} \eta=v_{\theta}$ where $\theta=\theta_{\eta}$. Since $\Gamma^{f} \subseteq \mathscr{F}$, and $v_{\theta}=v_{\phi}$ if and only if $\theta-\phi \in \Gamma^{f}$, we have $\operatorname{Ker} \bar{\delta}=\Gamma^{s c}+\Gamma^{f}$. Let $\Gamma(\mu)=\left\{\eta \in \Gamma:\left(\delta_{d} \eta\right) \mu=\mu\right\}$, i.e. such that $v_{\theta} \mu+d \theta=\mu$ where $\theta=\theta_{\eta}$. Then $\bar{\delta} \Gamma(\mu)=V(\mu)$.

Since $\mathscr{C}_{d}$ is abelain $V(\mu)$ and $\Gamma(\mu)$ depend only on the $\mathscr{C}_{d}$-orbit of $\mu$ in $\mathscr{F}_{d}$. Note that $V(\mu)=1$ in three particular cases, (i) $d=1$ with arbitrary $\Gamma$, (ii) $\Gamma^{e}=\Gamma^{s c}$ with arbitrary $d$, and (iii) $\mu=0$.

\section{Semi-simple classes.}

3.1. Automorphisms. Let $\psi$ be an algebraic automorphism of $G$ and assume $\psi$ has finite order, equal to $a$. We assume that $a$ is relatively prime to the characteristic of $k$. By $[14, \S 7]$ we may suppose that $\psi$ stabilizes a maximal torus $T$ and a Borel subgroup $B$ containing T. Thus $\psi$ induces a permutation $\beta \rightarrow \psi(\beta)$ on both $\Sigma$ and $\Pi$, defined by $\psi U_{\psi(\beta)}=U_{\beta}$ for $\beta \in \Sigma$.

If $\psi$ is multiplied by an inner automorphism, defined by a suitable element of $T$, we may suppose that $\psi\left(x_{\psi(\alpha)}(\zeta)\right)=x_{\alpha}(\zeta)$ for all $\zeta \in k^{*}$, $\pm \alpha \in \Pi$. From now on, we assume that $\psi$ is always in this "standard form" relative to $T$ and $B$.

The possible actions of $\psi$ on $G$ are easily described: if $\left\{E_{1}, \cdots, E_{s}\right\}$ is a $\psi$-orbit of simple components of $E=E(G)$ then $\psi^{s} E_{1}=E_{1}$ and $\psi^{s} \neq 1$ on $E_{1}$ only if $E_{1}$ is of type $A, D$, or $E_{6}$. The restriction of $\psi$ to $F=F(G)$ defines an element of $G L(f, Z) \simeq$ Aut $F$ where $f=\operatorname{dimension}$ of $F$.

Let $\langle G, \psi\rangle$ be the semi-direct product defined by $\psi g \psi^{-1}=\psi(g)$ and $\psi^{a}=1$. We wish to describe the classes of semi-simple elements of finite order in $\langle G, \psi\rangle$. It suffices to describe those classes in the coset $G \psi$ under conjugation by $G$.

In practice, $\psi=1$ is the most important example. Many of the following calculations simplify considerably in this case.

3.2. The root system $\Sigma_{\psi} \quad \psi$ induces endomorphisms on $X$ and $\Gamma$ (as in 2.3 but, for convenience, we use $\psi$ instead of $\psi_{*}$ ). The action on $X$ is consistent with the permutation of $\Sigma$. Put $C_{\psi}=\psi-1$ and $S_{\psi}=$ $1+\psi+\cdots+\psi^{a-1}$. Define $X_{c}=\left\{\chi \in X: C_{\psi} \chi=0\right\}, X_{s}=\left\{\chi \in X: S_{\psi} \chi=0\right\}$ and $\Gamma_{c}, \Gamma_{s}$ similarly. Note that $X_{c}$ and $\bar{\Gamma}=\Gamma / \Gamma_{s}$ are natural $Z$-duals via $\bar{\eta}(\chi) \equiv\left(\eta+\Gamma_{s}\right)(\chi)=\eta(\chi)$ for $\chi \in X_{c}$.

Let $T_{0}=\left\{t^{-1} \psi(t): t \in T\right\}$, then $T_{0}=\operatorname{Ann} X_{c}$ is a sub-torus of $T$. Put $\bar{T}=T / T_{0}$ then we have the natural isomorphisms $X(\bar{T}) \simeq X_{c}, \Gamma(\bar{T}) \simeq \bar{\Gamma}$.

If $n \in N$ define $\psi(w(n))=\psi(n) T$ and let $W_{\psi}=$ 
$\{w \in W: \psi(w)=w\} . \quad W_{\psi}$ acts as a reflection group on $\bar{T}$ and hence on $X_{c}, \bar{\Gamma}$ (see [14, §1] and below).

For $\alpha \in \Sigma$ let $0_{\alpha}=\{\alpha, \psi \alpha, \cdots\}$ denote the orbit due to the action of \%. Let $0,0^{\prime}$ be two such orbits in $\Sigma$, if $\alpha \in 0, \alpha^{\prime} \in 0^{\prime}$ and $\alpha+\alpha^{\prime} \in \Sigma$ define $0+0^{\prime}=0_{\alpha+\alpha^{\prime}}$. This sum is well-defined; i.e., it is independent of the choice of the representatives $\alpha, \alpha^{\prime}$. The orbit 0 is called 'good' if there does not exist an orbit $0^{\prime}$ such that $0^{\prime}+0^{\prime}=0$ ('bad' orbits can only occur if $E(G)$ contains components of type $A$ of even rank).

Let $0_{\alpha}$ be a good orbit, put

$$
A_{\alpha}= \begin{cases}2(\alpha+\psi \alpha+\cdots) & \text { if } 0_{\alpha}+0_{\alpha} \text { exists } \\ (\alpha+\psi \alpha+\cdots) & \text { otherwise }\end{cases}
$$

and then define $\Sigma_{\psi}=\left\{A_{\alpha}: \alpha \in \Sigma, 0_{\alpha}\right.$ a good orbit $\} . \quad \Sigma_{\psi}$ is a root-system in $X_{c}$ with $\hat{\alpha}+\Gamma_{s} \in \bar{\Gamma}$ the co-root corresponding to $A_{\alpha}$.

If $0_{\alpha}+0_{\alpha}$ exists, there is a unique $\alpha^{\prime}=\psi^{s} \alpha \in 0_{\alpha}$, where $\left|0_{\alpha}\right|=2 s$, such that $\alpha+\alpha^{\prime} \in \Sigma$. Define

$$
W_{A_{\alpha}}= \begin{cases}w_{\alpha+\alpha^{\prime}} \boldsymbol{w}_{\psi\left(\alpha+\alpha^{\prime}\right)} \cdots & \text { if } 0_{\alpha}+0_{\alpha} \text { exists } \\ w_{\alpha} w_{\psi \alpha} \cdots & \text { otherwise. }\end{cases}
$$

The products depend only on $0_{\alpha}$ and not on the order. From $[14, \S 1]$ we have $W_{\psi}=\left\langle w_{A}: A \in \Sigma_{\psi}\right\rangle$. Note that if $A=A_{\alpha}$ and $\chi \in X_{c}$ then $w_{A} \chi=$ $\chi-\hat{\alpha}(\chi)$. Let $\Pi_{\psi}=\left\{A_{\alpha} \in \Sigma_{\psi}: \alpha \in \Pi\right\}$ and $\Sigma_{\psi}^{+}=\left\{A_{\alpha} \in \Sigma_{\psi}: \alpha \in \Sigma^{+}\right\}$.

When $G$ is simple and $\psi \neq 1$ the type of $\Sigma_{\psi}$ is:

\begin{tabular}{|c|lllll|}
\hline$\Sigma$ & $A_{2 r}$ & $A_{2 r-1}$ & $D_{r}$ & $E_{6}$ & $D_{4}$ \\
\hline$\Sigma_{\psi}$ & $C_{r}$ & $B_{r}$ & $C_{r-1}$ & $F_{4}$ & $G_{2}$ \\
\hline$a$ & 2 & 2 & 2 & 2 & 3 \\
\hline
\end{tabular}

If $G=E(G)$ and $\Gamma=\Gamma^{s c}$ then $\bar{\Gamma}$ is spanned by the elements $\hat{\alpha}+\Gamma_{s}$ and hence is the 'simply-connected' lattice for $\bar{T}$ and $\Sigma_{\psi}$.

\subsection{Reduction to $\bar{T}$. If $t \in T$ let $\bar{t}=t T_{0} \in \bar{T}$.}

Proposition C. (i) Any semi-simple class in $G \psi$ intersects $T \psi$.

(ii) If $t, t^{\prime} \in T$ then $t \psi$ is conjugate to $t^{\prime} \psi$ under $G$ if and only if $w \bar{t}=\bar{t}^{\prime}$ for some $w \in W_{\psi}$. 
Proof. (i) Let $g \in G$ and suppose $g \psi$ is semi-simple. By $[\mathbf{1 4}, \S 7]$ $g \psi$ fixes $T^{x}$ and $B^{x}$ for some $x \in G$. Since $\psi$ stabilizes $T$ and $B$ and $\psi(x)=\psi x \psi^{-1}$ we have $x g \psi\left(x^{-1}\right) \in B \cap N$. Hence $x g \psi\left(x^{-1}\right)=t \in T$ and $g \psi=(t \psi)^{x}$.

(ii) We first show that if $w \in W_{\psi}$ then we can choose $n \in N$ so that $w=w(n)$ and $\psi n=n$. It suffices to do this for $w=w_{A}$ where $A \in \Pi_{\psi}$. For any $\alpha \in \Pi$ define $n_{\alpha}=x_{\alpha}(1) x_{-\alpha}(-1) x_{\alpha}(1)$. We may assume that $x_{\alpha}, x_{-\alpha}$ are chosen such that $n_{\alpha} \in N$. Then $w\left(n_{\alpha}\right)=w_{\alpha}$ and, since $\psi$ is in standard form, $\psi\left(n_{\psi \alpha}\right)=n_{\alpha}$. If $w_{A}=w_{\alpha} w_{\psi \alpha} \cdots$ put $n_{A}=$ $n_{\alpha} n_{\psi \alpha} \cdots$. In this case, if $\alpha, \beta$ are distinct elements in $0_{\alpha}$ then $\left[U_{\alpha}, U_{\beta}\right]=$ 1 and hence $\left[n_{\alpha}, n_{\beta}\right]=1$. Thus $\psi n_{A}=n_{A}$. If $w_{A}=w_{\alpha+\alpha^{\prime}} w_{\psi\left(\alpha+\alpha^{\prime}\right)} \cdots$ (the case where $0_{\alpha}+0_{\alpha}$ exists) let $n_{A}=\left(n_{\alpha} n_{\alpha^{\prime}} n_{\alpha}\right) \cdots$. Now a direct calculation shows that $n_{\alpha} n_{\alpha^{\prime}} n_{\alpha}=n_{\alpha^{\prime}} n_{\alpha} n_{\alpha^{\prime}}$ and since, as above, distinct terms in $n_{A}$ commute we again have $\psi n_{A}=n_{A}$.

To prove (ii) use the Bruhat normal form for the conjugating element. Thus, in an obvious notation, (usnv)t $\psi=t^{\prime} \psi(u s n v)$. This yields $w(n) \in W_{\psi}$ and by the previous paragraph we may now suppose that $\psi n=n$. Thus, if $w=w(n), w t=t^{\prime} s^{-1} \psi(s)$ and hence $w \bar{t}=\bar{t}^{\prime}$.

3.4. Computation of the classes. Let $\mathscr{K}$ be a class of semi-simple elements of order $d$ in $G \psi$. Thus $d$ is a multiple of $a$ (= order of $\psi$ ) and is also relatively prime to the characteristic of $k$. These are the only restrictions on $d$. Choose $\xi_{d} \in k^{*}$ a fixed primitive $d$ th root of unity.

Let $T_{d}=\left\{t \in T:(t \psi)^{d}=1\right\}$ and put $\bar{T}_{d}$ for its image in $\bar{T}$. Let $\bar{T}_{(d)}=\left\{\bar{t} \in \bar{T}: \bar{t}^{d}=1\right\}$ and note that $\bar{T}_{d} \subseteq \bar{T}_{(d)}$.

For $\bar{\mu} \in \bar{\Gamma}$ define $\bar{\mu}\left(\xi_{d}\right) \in \bar{T}$ by $\bar{\mu}\left(\xi_{d}\right)=\overline{\mu\left(\xi_{d}\right)}\left(\equiv \mu\left(\xi_{d}\right) T_{0}\right)$ where $\mu \in \Gamma$ is any inverse image of $\bar{\mu}$. The map $\bar{\mu} \rightarrow \bar{\mu}\left(\xi_{d}\right)$ is a homomorphism of $\bar{\Gamma}$ onto $\bar{T}_{(d)}$ with kernel $d \bar{\Gamma}$. Let $\bar{\Gamma}_{c}$ denote the image of $\Gamma_{c}$ in $\bar{\Gamma}$.

\section{Proposition D. The homomorphism $\bar{\mu} \rightarrow \bar{\mu}\left(\xi_{d}\right)$ maps $\bar{\Gamma}_{c}$ onto $\bar{T}_{d}$.}

Proof. Let $\mu \in \Gamma_{c}$ and $e=d / a$ then $\left(\mu\left(\xi_{d}\right) \psi\right)^{d}=e S_{\psi} \mu\left(\xi_{d}\right)=$ $d \mu\left(\xi_{d}\right)=1$ and hence $\bar{\mu}\left(\xi_{d}\right) \in \bar{T}_{d}$. Conversely, suppose $\mu\left(\xi_{d}\right) \in T_{d}$ then, as above, $e S_{\psi} \mu \in d \Gamma$. Thus $e S_{\psi} \mu=d \lambda$ some $\lambda \in \Gamma$. Since $\psi S_{\psi}=S_{\psi} \psi$ we have $\lambda \in \Gamma_{c}$ and hence $d \lambda=e S_{\psi} \lambda$. Therefore $\mu-\lambda \in \Gamma_{s}$, as required.

Thus the semi-simple classes, of order dividing $d$, in $G \psi$ are in $1: 1$ correspondence with the orbits of $\mathscr{A}_{d}\left(W_{\psi}, \bar{\Gamma}\right)$ on $\bar{\Gamma}_{c}$. Using proposition A these orbits are easily found.

We have $\mathscr{F}_{d}(\bar{\Gamma})=\left\{\bar{\mu} \in \bar{\Gamma}: 0 \leqq \bar{\mu}\left(A_{\alpha}\right) \leqq d\right.$ all $\left.A_{\alpha} \in \Sigma_{\psi}^{+}\right\}$. If $\{\bar{\mu}, \cdots\}$ is a $\mathscr{C}_{d}(\bar{\Gamma})$-orbit in $\mathscr{F}_{d}(\bar{\Gamma}) \cap \bar{\Gamma}_{c}$ let $\mu \in \Gamma_{c}$ be an inverse image of $\bar{\mu}$. A representative for the class corresponding to $\{\bar{\mu}, \cdots\}$ is $\mu\left(\xi_{d}\right) \psi$. Since $\Gamma_{s} \cap \Gamma_{c}=0 \mu$ is uniquely determined by $\bar{\mu}$; also $\left[\mu\left(\xi_{d}\right), \psi\right]=1$. 
3.5. Examples. Let $G$ be simple of type $D_{r}$, with $r \geqq 4$ and even, and suppose $X=X^{a d}$. Let $\psi$ be of order $a=2$.

Index the simple roots $\Pi=\left\{\alpha_{1}, \cdots, \alpha_{r}\right\}$ as follows,

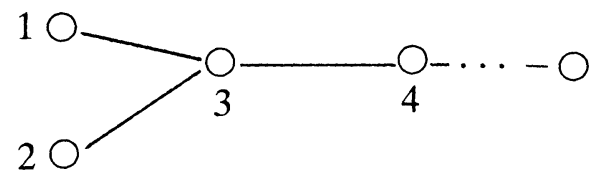

Let $\left\{\eta_{j}\right\} \in \Gamma$ be a dual basis to $\Pi$, i.e. $\eta_{j}\left(\alpha_{i}\right)=\delta_{i j}$. We have $\psi \alpha_{1}=\alpha_{2}$, $\psi \alpha_{2}=\alpha_{1}, \psi \alpha_{i}=\alpha_{\imath}$ for $i \geqq 3$ and similarly for $\psi \eta_{i}$. Thus $\Gamma_{s}=\left\langle\eta_{1}-\eta_{2}\right\rangle$, $\Gamma_{c}=\left\langle\eta_{1}+\eta_{2}, \eta_{3}, \cdots, \eta_{r}\right\rangle \quad$ and $\quad$ so $\quad \bar{\Gamma}=\left\langle\bar{\eta}_{1}, \bar{\eta}_{3}, \cdots, \bar{\eta}_{r}\right\rangle$ and $\bar{\Gamma}_{c}=$ $\left\langle 2 \bar{\eta}_{1}, \bar{\eta}_{3}, \cdots, \bar{\eta}_{r}\right\rangle . \quad \Pi_{\psi}=\left\{\alpha_{1}+\alpha_{2}, \alpha_{3}, \cdots, \alpha_{r}\right\} \quad$ and $\quad A_{*}=$ $-\left(\alpha_{1}+\alpha_{2}+2 \alpha_{3}+\cdots+2 \alpha_{r}\right)$ is the corresponding low root in $\Sigma_{\psi \cdot}$. Thus $\mathscr{F}(\bar{\Gamma})=\left\{0, \bar{\eta}_{1}\right\}$ and so $\mathscr{C}_{d}(\bar{\Gamma})=\left\{1, a\left(v, d \bar{\eta}_{1}\right)\right\}$ where $v \in V_{\psi}$ (see 4.2), is defined by $v \bar{\eta}_{1}=-\bar{\eta}_{1}, v \bar{\eta}_{j}=-2 \bar{\eta}_{1}+\bar{\eta}_{r+3-j}$ for $3 \leqq j \leqq r$.

Consider the case $d=2$. Proposition A gives the following $\mathscr{C}_{2}(\bar{\Gamma})$ orbits in $\mathscr{F}_{2}(\bar{\Gamma}):\left\{0,2 \bar{\eta}_{1}\right\},\left\{\bar{\eta}_{1}\right\},\left\{\bar{\eta}_{3}, \bar{\eta}_{r}\right\},\left\{\bar{\eta}_{4}, \bar{\eta}_{r-1}\right\}, \cdots,\left\{\bar{\eta}_{p}, \bar{\eta}_{p+1}\right\}$ where $p=$ $(r+2) / 2$. Except for $\left\{\bar{\eta}_{1}\right\}$, all lie in $\bar{\Gamma}_{c}$. Thus there are $r / 2$ classes of involutions in $G \psi$. Representative elements are $\psi, \eta_{3}(-1) \psi$, $\eta_{4}(-1) \psi, \cdots, \eta_{p}(-1) \psi$.

If $r=4$ and $\psi$ is of order 3 a similar calculation with $d=3$ gives two classes, with representatives $\psi, \eta_{3}\left(\xi_{3}\right) \psi$.

Calculations for other $G, \psi$ are entirely similar. The only requirement is a practical description of $\Pi, \Pi^{*}, X, \Gamma$ and $V$.

\section{Centralizer subgroups.}

4.1. The component of the identity. We continue with the notation introduced in $\$ 3$. Let $\mu \in \Gamma_{c}$ and $\bar{\mu} \in \mathscr{F}_{d}(\bar{\Gamma})$ then $\varphi=\mu\left(\xi_{d}\right) \psi$ is a typical semi-simple element in $G \psi$ with order dividing $d$. We put $C=C_{G}(\varphi)$ and will begin by describing the structure of $C^{0}$. From [14, $\S 8]$ we know it is reductive (see also our proof of proposition $E$ ).

Put $T_{\psi}=\{t \in T: \psi t=t\}$ then $T_{\psi}^{0}$ is a maximal torus of $C^{0}$. Since $T_{\psi}^{0}=$ Ann $X_{s}$ we have the isomorphisms $X\left(T_{\psi}^{0}\right) \simeq X / X_{s}=\bar{X}$ and $\Gamma\left(T_{\psi}^{0}\right) \simeq$ $\Gamma_{c}$. If $\alpha \in \Sigma$ let $\bar{\alpha}$ denote its image in $\bar{X}$ and put $\Sigma(\varphi)=$ $\left\{\bar{\alpha}: \mu\left(A_{\alpha}\right) \in d Z, A_{\alpha} \in \Sigma_{\psi}\right\}$.

\section{Proposition E. $\Sigma(\varphi)$ is a root system for $C^{0}$ in $\bar{X}$.}

Proof. Let $\alpha \in \Sigma$ and $0_{\alpha}$ a good orbit (see 3.2) and suppose first that $0_{\alpha}+0_{\alpha}$ does not exist. Choose $x_{\alpha}$ and then define $x_{\psi \alpha}, x_{\psi^{2} \alpha}, \cdots$ by $\psi\left(x_{\psi^{i} \alpha}\right)=x_{\psi^{\prime-1} \alpha}$ for $i=1,2, \cdots, s-1$ where $s=\left|0_{\alpha}\right|$. A simple argument, using induction on the height of $\alpha$ and the fact that $\psi$ is in standard form, shows that $\psi x_{\alpha}=x_{\psi^{s-1} \alpha}$. This is the key-point. Since 
$(\alpha+\psi \alpha+\cdots) \mu\left(\xi_{d}\right)=\xi_{d}^{\mu\left(A_{\alpha}\right)}$ we now find that

$$
C \cap\left(U_{\alpha} U_{\psi \alpha} \cdots\right)=\left\{\begin{array}{lll}
1 & \text { if } & \mu\left(A_{\alpha}\right) \notin d Z \\
U_{\bar{\alpha}} & \text { if } & \mu\left(A_{\alpha}\right) \in d Z
\end{array}\right.
$$

where $U_{\tilde{\alpha}}=x_{\bar{\alpha}}\left(k_{+}\right)$and $x_{\tilde{\alpha}}(\zeta)=x_{\alpha}(\zeta) x_{\psi \alpha}\left(\epsilon_{1} \zeta\right) \cdots x_{\psi^{s-1} \alpha}\left(\epsilon_{s-1} \zeta\right)$ for $\zeta \in k$ and $\epsilon_{i}=\xi_{d}^{-e_{i}}$ with $e_{i}=\mu\left(\alpha+\psi \alpha+\cdots+\psi^{i-1} \alpha\right)$. If $t \in T_{\psi}^{0}$ then $\alpha(t)=$ $\psi \alpha(t)=\cdots=\bar{\alpha}(t)$, hence $t x_{\tilde{\alpha}}(\zeta) t^{-1}=x_{\tilde{\alpha}}(\zeta \bar{\alpha}(t))$ and so $\bar{\alpha}$ is a root.

If $0_{\alpha}+0_{\alpha}$ exists the argument is completely similar but slightly more involved. The case where $G$ is simple of type $A_{2}$ and $\psi \neq 1$ is quite typical. In this case suppose $\Pi=\{\alpha, \beta\}$ and $\psi \neq 1$. Using the commutation rules in $U$ and the fact that $\psi$ is in standard form we get

$$
\psi\left(x_{\alpha}\left(\zeta_{\alpha}\right) x_{\beta}\left(\zeta_{\beta}\right) x_{\alpha+\beta}(\zeta)\right)=x_{\alpha}\left(\zeta_{\beta}\right) x_{\beta}\left(\zeta_{\alpha}\right) x_{\alpha+\beta}\left(\epsilon \zeta_{\alpha} \zeta_{\beta}-\zeta\right)
$$

where $\epsilon= \pm 1$.

Since $\mu\left(A_{\alpha}\right)=\mu(2(\alpha+\beta))$ we have $\xi_{d}^{\mu(\alpha+\beta)}= \pm 1$ for $\mu\left(A_{\alpha}\right) \in d Z$. Thus

$$
C \cap U=\left\{\begin{array}{lll}
1 & \text { if } & \mu\left(A_{\alpha}\right) \notin d Z \\
U_{\bar{\alpha}} & \text { if } & \mu\left(A_{\alpha}\right) \in d Z
\end{array}\right.
$$

where the form of $U_{\bar{\alpha}}=x_{\bar{\alpha}}\left(k_{+}\right)$depends on whether or not $\mu\left(A_{\alpha}\right) \in$ $2 d Z$. We find $x_{\bar{\alpha}}(\zeta)=x_{\alpha+\beta}(\zeta)$ if $\mu\left(A_{\alpha}\right) \notin 2 d Z$ and $x_{\bar{\alpha}}(\zeta)=$ $x_{\alpha}(\zeta) x_{\beta}\left(\xi_{d}^{\mu(\alpha)} \zeta\right) x_{\alpha+\beta}\left(\epsilon \xi_{d}^{\mu(\alpha)} \zeta^{2} / 2\right)$ if $\mu\left(A_{\alpha}\right) \in 2 d Z$.

The co-root in $\Gamma_{c}$ corresponding to $\bar{\alpha} \in \Sigma(\varphi)$ is denoted by $\hat{A}_{\alpha}$ and is given by replacing the $\alpha$ 's by $\hat{\alpha}$ 's in the definition of $A_{\alpha}$.

Using the Bruhat normal form in $G$ we have $C=\langle C \cap U, C \cap N\rangle$ where $U$ is the unipotent radical of $B$. Thus the above proof shows that $C^{0}=\left\langle U_{\bar{\alpha}}, T_{\psi}^{0}: \bar{\alpha} \in \Sigma(\varphi)\right\rangle$. Hence its Weyl group is $\left(C^{0} \cap N\right) / T_{\psi}^{0} \simeq$ $\left\langle w_{A}: \mu(A) \in d Z, A \in \Sigma_{\psi}\right\rangle$.

The structure of $C^{0}$ may be described by the methods of 2.1 and 2.2 using $\bar{X}, \Gamma_{c}, \Sigma(\varphi)$ in place of $X, \Gamma, \Sigma$. The fact that $\left\{\bar{\alpha} \in \Sigma(\varphi): A_{\alpha} \in \Pi_{\psi}^{*}\right\}$ contains a set of simple roots for $\Sigma(\varphi)$ is very useful in calculations.

4.2. Structure of $C / C^{0}$. We will use proposition $B$ but with $W, \Gamma$ replaced by $W_{\psi}, \bar{\Gamma}$. Thus $V_{\psi}$ will denote the stabilizer in $W_{\psi}$ of the extended root system $\Pi_{\psi}^{*}$ corresponding to $\Pi_{\psi}$ in $\Sigma_{\psi}$.

Observe that $C=C^{0}(C \cap N)$ and if $n \in C \cap N$ then $w(n) \in$ $W_{\psi}$. Let $\epsilon$ denote the homomorphism of $C \cap N$ into $W_{\psi}$ given by $\epsilon(n)=w(n)$. A simple calculation, using the proof of part (ii) of 
proposition $C$, yields that $\operatorname{Ker} \epsilon=T_{\psi}$ and $\operatorname{Im} \epsilon=W_{\psi}(\bar{\mu})$. Now the results of 4.1 together with proposition B imply that $\epsilon\left(C^{0} \cap N\right)=W_{\psi}(\bar{\mu})^{s c}$ and hence $(C \cap N) / T_{\psi}\left(C^{0} \cap N\right) \simeq V_{\psi}(\bar{\mu})$.

Since $T_{\psi}=$ Ann $C_{\psi} X$ and $Z(G)=$ Ann $X^{a d}$ we have $T_{\psi} \cap Z(G)=$ $\operatorname{Ann}\left(C_{\psi} X+X^{a d}\right)$ and thus $T_{\psi}^{0}\left(T_{\psi} \cap Z(G)\right)=\operatorname{Ann}\left(X_{s} \cap\left(C_{\psi} X+X^{a d}\right)\right)$. Since $\psi$ permutes the simple roots $\Pi$ and $X^{a d}=Z \Pi$ we have $C_{\psi} X^{a d}=$ $X^{a d} \cap X_{s}$ and hence find that $T_{\psi}^{0}\left(T_{\psi} \cap Z(G)\right)=T_{\psi}$. Thus,

Proposition F. (i) $C=C^{1}(C \cap N)$ where $C^{1}=C^{0}\left(T_{\psi} \cap Z(G)\right)$.

(ii) $C / C^{1} \simeq V_{\psi}(\bar{\mu})$ and $C^{1} / C^{0} \simeq X_{s} / C_{\psi} X$.

In particular $\left|C / C^{0}\right|$ is finite and relatively prime to the characteristic of $k$. If $\psi=1$ then $C^{1}=C^{0}$ and $C / C^{0} \simeq V(\mu) \simeq \Gamma(\mu) / \Gamma^{s c}+\Gamma^{f}$, as in 2.4 .

The action of $C / C^{0}$ on $C^{0}$ is found from the permutations induced on $\Sigma(\varphi)$ by $V_{\psi}(\bar{\mu})$. In general $C$ will not split over $C^{0}$, however in certain cases it does: an example is $G$ simple, $X=X^{a d}, \psi=1$ and $d=2$. We will not prove this result but the example below should indicate the line of argument.

4.3. Examples. Suppose $G$ is simple of type $D_{4}, X=X^{a d}$ and $\psi=1$. We use the notation in 3.5 and consider the involution $\eta_{3}(-1)$ in $G$. We wish to describe the structure of $C=C_{G}\left(\eta_{3}(-1)\right)$.

Since $d=2$ and $\alpha_{*}=-\left(\alpha_{1}+\alpha_{2}+\alpha_{4}+2 \alpha_{3}\right)$ we find $\Pi^{*} \cap$ $\Sigma\left(\eta_{3}(-1)\right)=\left\{\alpha_{1}, \alpha_{2}, \alpha_{4}, \alpha_{*}\right\}$ as a set of simple roots for $C^{0}$. Since $-\alpha_{3}=\left(\alpha_{1}+\alpha_{2}+\alpha_{4}+\alpha_{*}\right) / 2$ we see that $C^{0}$ is a central product of four $S L_{2}(k)$ 's (and $Z\left(C^{0}\right)=\left\langle\eta_{3}(-1)\right\rangle$ ).

In $D_{4} ; \mathscr{F}=\left\{0, \eta_{1}, \eta_{2}, \eta_{4}\right\}$ and if $v_{l}=v_{\eta_{i}}$ we have $v_{i} \eta_{3}=-2 \eta_{i}+\eta_{3}$ for $i=1,2,4$. Thus $V\left(\eta_{3}\right)=V$ and hence $C / C^{0} \simeq Z_{2} \times Z_{2}$ and acts as a regular permutation group on the four components of $C^{0}$.

If $n_{1}$ lies in the coset $v_{1} \in W$, it induces an algebraic (outer) automorphism of $C^{0}$ and we may choose $n_{1}$ so that it is in standard form (see 3.1) relative to the simple roots $\left\{\alpha_{1}, \alpha_{2}, \alpha_{4}, \alpha_{*}\right\}$. Thus $n_{1}^{2}$ will centralize $C^{0}$. Now $n_{1} x_{3}(\zeta) n_{1}^{-1}=x_{3}( \pm \zeta)$ and hence $n_{1}^{2}$ centralizes $U_{\alpha_{3}}$. Since $\left\langle C^{0}, U_{\alpha_{3}}\right\rangle=G$ we conclude that $n_{1}^{2}=1$. Choose $n_{2}$ similarly, so that $n_{2}^{2}=1$, and put $n_{4}=n_{1} n_{2}$. Since $n_{1}, n_{2}$ are in standard form so also is $n_{4}$ and thus $n_{4}^{2}=1$. This implies $n_{1} n_{2}=n_{2} n_{1}$ and so $\left\langle n_{1}, n_{2}\right\rangle$ is a complement to $C^{0}$ in $C$ and the extension splits.

As a further example we describe the structure of $C$ when $G$ is simple, $X=X^{a d}, \psi \neq 1$ and where $\varphi$ runs over the classes of order $d=a$ in the coset $G \psi$. A typical calculation of these classes was given in 3.5. We find that $C^{0}$ is always semi-simple; the following table describes the various cases. 


\begin{tabular}{|l|l|c|}
\hline$G$ & $a$ & $C^{0}$ \\
\hline$A_{2 r}$ & 2 & $B_{r}$ \\
\hline$A_{2 r-1}, r \geqq 2$ & 2 & $C_{r} D_{r}$ \\
\hline$D_{r} \quad r \geqq 4$ & 2 & $B_{r-1}, B_{i} \times B_{r-1-i} \quad$ for $\quad i=1,2, \cdots,\left[\frac{r-1}{2}\right]$ \\
\hline$D_{4}$ & 3 & $G_{2}, A_{2}$ \\
\hline$E_{6}$ & 2 & $F_{4}, C_{4}$ \\
\hline
\end{tabular}

The lattice for $C^{0}$ is always adjoint and $C=C^{0}$ with two exceptions: (i) $G=A_{2 r-1}, C^{0}=D_{r}$ when $C=\left\langle C^{0}, n\right\rangle$ where $n$ is the automorphism interchanging $\alpha_{1}$ and $\alpha_{2}$ and $n^{2}=1$, (ii) $G=D_{r} \quad r=$ odd, $C^{0}=$ $B_{(r-1) / 2} \times B_{(r-1) / 2}$ when $C=\left\langle C^{0}, n\right\rangle$ where $n$ interchanges the two components and $n^{2}=1$.

\section{The finite groups.}

5.1. Endomorphisms of finite type. Let $\sigma$ be a morphism of $G$ onto itself with $\operatorname{ker} \sigma=1$ and such that $G(\sigma)=\{g \in G: \sigma g=g\}$ is finite. If $E(G) \neq 1$ the existence of such a $\sigma$ implies that the characteristics of $k$ is $p \neq 0$ and that $\sigma^{-1}$ is not a morphism (although $\sigma$ is an automorphism of $G$ considered as an abstract group). The possibilities for $\sigma$ are well known, see [14, §11].

We may suppose that $\sigma$ stabilizes $T$ and hence that $\sigma$ induces a permutation on $\Sigma$. This permutation is consistent with the action of $\sigma_{*}$ on $X$ as defined in 2.3. If $n \in N$ and $\rho=i_{n} \sigma$ then $\rho_{*}=\sigma_{*} w^{-1}$ where $w=w(n)($ and $\rho=w \sigma$ on $\Gamma)$. Using a theorem of Lang [11] we may find $h \in G$ such that $h \sigma\left(h^{-1}\right)=n$ and hence $g \rightarrow i_{h} g$ gives an isomorphism of $G(\sigma)$ onto $G(\rho)$.

5.2. Structure of $G(\sigma)$. Put $L=0^{p^{\prime}}(G(\sigma))$ where $p$ is the characteristic of $k .0^{p^{\prime}}(X)$ denotes the normal subgroup of $X$ generated by all elements of order $p$. Thus $L$ is that subgroup of $G(\sigma)$ generated by all the unipotent elements. Put $J=C_{G(\sigma)}(L), Z=J \cap L$ and $D=G(\sigma) / J L$. Then $J=Z(G(\sigma))$ and $Z=Z(L)$ and $D$ induces 'diagonal type' automorphisms of $L$. If we make allowance for the usual 8 exceptions $\left(A_{1}(2),{ }^{2} A_{2}(2), C_{2}(2),{ }^{2} C_{2}(2), G_{2}(2),{ }^{2} F_{4}(2)\right.$ and $\left.A_{1}(3),{ }^{2} G_{2}(3)\right)$ then $L$ is the layer of $G(\sigma)$, i.e., $L / Z$ is a direct product of simple components. 
The components in $L / Z$ can be immediately described once the action of $\sigma$ on $\Sigma$ (or on $\Pi$ ) is given. To describe $J$ we introduce $\tau_{*}=\sigma_{*}-1$ and note that it is always nonsingular on $X$. Then $T(\sigma)=$ $\{t \in T: \sigma t=t\}=$ Ann $\tau_{*} X$. Since $C_{G}(L)=C_{G}(E(G))$ we have $J=$ $\operatorname{Ann}\left(\tau_{*} X+X^{a d}\right)$ and hence $J \simeq X / \tau_{*} X+X^{a d}$.

We now describe $Z$ and $D$. Let $E^{s c}$ be a simply-connected covering group of $E=E(G)$ and let $\pi$ be the natural isogeny of $E^{s c}$ onto $E$. Choose $T^{s c}$ a maximal torus of $E^{s c}$ so that $\pi T^{s c}=T \cap E$. Let $\pi_{*}$ denote the natural homomorphism of $X$ into $X^{s c}=X\left(T^{s c}\right)$ induced by $\pi$. We have $\operatorname{Ker} \pi_{*}=X^{f}$ and $\operatorname{Ker} \pi=\operatorname{Ann}\left(\operatorname{Im} \pi_{*}\right)$. Define $\Delta^{s c}=$ $\Delta^{s c}(G)=X^{s c} / \pi_{*} X^{a d}$; this is a finite group, in fact just the 'weight lattice/root lattice' for the root system $\Sigma$.

We may extend $\sigma$ to $E^{s c}$ so that $\sigma \pi=\pi \sigma$ (see $\left.[14, \S 9]\right)$. Hence $\tau_{*}$ is defined on $X^{s c}$ and so on $\Delta^{s c}$. We use the notation $\operatorname{Ker} \tau_{*}, \operatorname{Im} \tau_{*}$ to denote the kernel and image for the action of $\tau_{*}$ on $\Delta^{s c}$. Let $\Delta, \Delta^{e}$ denote the images of $X, X^{e}$ respectively in $\Delta^{s c}$.

Proposition G. $Z \simeq \Delta / \Delta \cap \operatorname{Im} \tau_{*}$ and $D \simeq \operatorname{Ker} \tau_{*} / \Delta^{e} \cap \operatorname{Ker} \tau_{*}$.

Proof. $T \cap L=\pi\left(T^{s c}(\sigma)\right)$ and so $T \cap L=$ Ann $Y$ where $Y=$ $\pi_{*}^{-1}\left(\tau_{*} X^{s c} \cap \pi_{*} X\right)$. Thus, using $\tau_{*} X \subseteq Y$, we find $Z=J \cap(T \cap L)=$ $\operatorname{Ann}\left(Y+X^{a d}\right)$ and $J(T \cap L)=\operatorname{Ann}\left(\tau_{*} X+X^{a d} \cap Y\right)$. Hence

$$
Z \simeq X /\left(Y+X^{a d}\right)
$$

and

$$
D \simeq\left(\tau_{*} X+X^{a d} \cap Y\right) / \tau_{*} X \simeq\left(X^{a d} \cap Y\right) /\left(X^{a d} \cap \tau_{*} X\right) .
$$

Now $\operatorname{Im} \tau_{*}=\left(\tau_{*} X^{s c}+\pi_{*} X^{a d}\right) / \pi_{*} X^{a d}$ and, since $\tau_{*}$ is nonsingular on $X^{s c}$, Ker $\tau_{*}=\left(X^{s c} \cap \tau_{*}^{-1} \pi_{*} X^{a d}\right) / \pi_{*} X^{a d}$. Using the above expressions for $Z$ and $D$ and the fact that $X \cap \tau_{*}^{-1} X^{a d}=X^{e} \cap \tau_{*}^{-1} X^{a d}$ we immediately obtain the required formulas.

In practice the action of $\tau_{*}$ on $\Delta^{s c}$ is easily found and the above expression for $J, Z, D$ give a most useful and easy method for describing $G(\sigma)$; see for example [5]. To find the explicit extension of $G(\sigma)$ over $J L$ use the description of $J(T \cap L)$ given in the proof. Note that $\Delta^{s c}$ may be replaced by $0_{p^{\prime}}\left(\Delta^{s c}\right)$.

The above results extend to the disconnected case as follows: suppose $G^{+}=\left\langle G, \psi_{1}, \psi_{2}, \cdots\right\rangle$ where the $\psi_{i}$ are automorphisms of $G$. If $\sigma$ acts on $G^{+}$it permutes the cosets $G \psi_{i}$. Using [11] we see that $G^{+}(\sigma) \cap G \psi \neq \phi$ if and only if $\sigma$ fixes $G \psi$. 
5.3. Classes and centralizers in $G(\sigma)$. Let $\psi$ be as in 3.1 and put $G^{+}=\langle G, \psi\rangle$. We suppose that $\sigma$ is as described above and acts on $G^{+}$. We also assume that $\sigma(\psi)=\psi$. This is a minor restriction which is justified by the applications (by putting $\psi$ and $\sigma$ in standard form we may always assume $\sigma(\psi)=z \psi$, where $z \in Z(G)$; it is not difficult to extend the following arguments to this case). Using the results of $\S 3$ and $\S 4$ together with Lang's theorem see [13, §2, 3], we will now describe the semi-simple classes in $G^{+}(\sigma)$, under conjugation by $G(\sigma)$, and the structure of their corresponding centralizer subgroups in $G(\sigma)$.

Let $\mathscr{K}$ be as in 3.4 and suppose $\mu\left(\xi_{d}\right) \psi \in \mathscr{K}$ where $\mu \in \Gamma_{c}$ and $\bar{\mu} \in \mathscr{F}_{d}(\bar{\Gamma})$. For convenience, we put $\mathscr{K}=[\mu]$, with the understanding that $\psi, d, \xi_{d}$ are fixed.

Now $[\mu] \cap G^{+}(\sigma) \neq \phi$ if and only if $\sigma[\mu]=[\mu]$, which is equivalent to $\sigma \bar{\mu}$ and $\bar{\mu}$ lying in the same $\mathscr{A}_{d}\left(W_{\psi}, \bar{\Gamma}\right)$ orbit. The proof that $\mathscr{F}_{d}$ is a fundamental domain for $\mathscr{A}_{d}^{s c}$ yields a practical algorithm for finding the representative in $\mathscr{F}_{d}$ of any $\mathscr{A}_{d}^{s c}$ orbit (see Appendix 2). Thus proposition A will determine whether $[\mu]$ intersects $G^{+}(\sigma)$. Suppose now that $G^{+}(\sigma) \cap[\mu] \neq \phi$, then the algorithm yields a $w \in W_{\psi}$ such that $(w \sigma-1) \bar{\mu} \in d \bar{\Gamma}$. Let $w=n T$ and put $\rho=i_{n} \sigma$. Thus $(\rho-1) \bar{\mu} \in d \bar{\Gamma}$ which, since $d \Gamma \cap \Gamma_{c}=d \Gamma_{c}$, is equivalent to $\mu\left(\xi_{d}\right) \in G(\rho)$ and hence $\mu\left(\xi_{d}\right) \psi \in G^{+}(\rho)$.

Assume $\mu\left(\xi_{d}\right) \in G(\rho)$. The set $[\mu] \cap G^{+}(\rho)$ will, in general, split into several classes under conjugation by $G(\rho)$. Let $C=C_{G}\left(\mu\left(\xi_{d}\right) \psi\right)$ and put $[C, \rho]=\left\{c^{-1} \rho(c): c \in C\right\}$. By $[11][C, \rho] \supseteq C^{0}$ and hence $[C, \rho]$ is a group and the quotient $\tilde{C}=C /[C, \rho]$ is finite and abelian. Choose $\tilde{c} \in \tilde{C}$, then by 4.2 we may find $m \in \tilde{c} \cap N$ and may choose $h \in G$ such that $h^{-1} \rho(h)=m$. Then, again using [11], the $G(\rho)$ classes in $[\mu] \cap$ $G^{+}(\rho)$ are in $1: 1$ correspondence with the elements of $\tilde{C}: \tilde{c}$ corresponds to the class containing $h \mu\left(\xi_{d}\right) \psi h^{-1}$. It is easily checked that this correspondence is independent of the choices of $m, h$. Note that $h \mu\left(\xi_{d}\right) \psi h^{-1} \in G^{+}(\rho)$ since $\rho(h)=h m$ and $m \in C$.

In practice it is not necessary to compute $m, h$. Instead, define $\rho_{m}=i_{m} \rho$ then, since $i_{h}\left(G\left(\rho_{m}\right)\right)=G(\rho)$ we may replace $h \mu\left(\xi_{d}\right) \psi h^{-1} \in G(\rho)$ by $\mu\left(\xi_{d}\right) \psi \in G\left(\rho_{m}\right)$. Thus we describe the $G(\rho)$ classes in $[\mu] \cap G^{+}(\rho)$ by the pairs $[\mu, \rho],\left[\mu, \rho_{m}\right], \cdots$ where $m$ runs over coset representatives for $[C, \rho]$ in $C$.

If $\left[\mu, \rho_{m}\right]$ is such a class the centralizer in $G(\rho)$ of an element of this class is isomorphic to $C\left(\rho_{m}\right)$ (where $C=C_{G}\left(\mu\left(\xi_{d}\right) \psi\right)$ ). The structure of $C\left(\rho_{m}\right)$ is determined by the methods of 5.2. Note that we only need $w(m)$ for these calculations.

5.4. The semi-simple case. Throughout this subsection we assume that $G$ is semi-simple. Thus $X^{e}=X, X^{f}=0$, etc. We introduce the symbol $G(\mu)^{u}$ in place of $0^{p^{\prime}}(G(\sigma))=L$ of 5.2. $G(\sigma)^{u}$ is generated by 
the unipotent elements ( $=p$-elements) in $G(\sigma)$. If $X=X^{a d}$ and $G$ is simple then $G(\sigma)^{u}$ is, with the usual 8 exceptions, a non-abelian simple group.

The expression for $J$ in 5.2 may be simplified to $J \simeq$ $\Delta / \tau_{*} \Delta$. Together with $\Delta^{e}=\Delta$ and the formula for $D$ this yields $|G(\sigma)|=\left|G(\sigma)^{u}\right| Z|\cdot| \operatorname{Ker} \tau_{*} \mid$. In particular, $|G(\sigma)|$ depends only on $\Sigma$ and $\sigma$ and not on $X$.

We now consider two questions: first, if $[\mu]$ is a semi-simple class in $G$ and $G(\sigma) \cap[\mu] \neq \phi$ when is $G(\sigma)^{u} \cap[\mu] \neq \phi$ ? and second, if $C=C_{G}\left(\mu\left(\xi_{d}\right)\right)$ what is the structure of $C \cap G(\sigma)^{u}$ ? We have phrased these questions for the case $\psi=1$ but their extension to $\psi \neq 1$ is quite obvious.

To answer the first question we may suppose $\mu \in \mathscr{F}_{d}$ and $(\sigma-1) \mu \in$ $d \Gamma$, and hence $\mu\left(\xi_{d}\right) \in G(\sigma)$. Then,

Proposition H. $\mu\left(\xi_{d}\right) \in G(\sigma)^{u}$ if and only if $(1 / d)(\sigma-1) \mu \in$ $(\sigma-1) \Gamma+\Gamma^{s c}$.

Proof. Let $\mathscr{S}=\{\mu-d \theta: \theta \in \mathscr{F}\}$. By proposition A the set $\mathscr{S}$ contains a set of representatives for those $\mathscr{A}_{d}^{s c}$ orbits which lie in the $\mathscr{A}_{d}$ orbit containing $\mu$. There is a positive integer $e$ such that $e \eta \in \Gamma^{s c}$ for all $\eta \in \mathscr{S}$. Choose $\zeta \in k^{*}$ a primitive $(e d)$ th root of unity such that $\zeta^{e}=\xi_{d}$. Let $G^{s c}$ be the simply connected covering group of $G$ and $\pi$ the isogeny of $G^{s c}$ onto $G$. We may identify $\Gamma^{s c}$ with the $\Gamma$-lattice for $G^{s c}$. Then since $\operatorname{ker} \pi=\{e d \theta(\zeta): \theta \in \mathscr{F}(\Gamma)\}$ we have $\pi^{-1}\left(\mu\left(\xi_{d}\right)\right)=$ $\{e \eta(\zeta): \eta \in \mathscr{P}\} \subseteq G^{s c}$.

Since $\pi\left(G^{s c}(\sigma)\right)=G(\sigma)^{u}$ we see that $\mu\left(\xi_{d}\right) \in G(\sigma)^{u}$ if and only if $e \eta(\zeta) \in G^{s c}(\sigma)$ for some $\eta \in \mathscr{S}$, i.e., if and only if $(\sigma-1) e(\mu-d \theta) \in$ $e d \Gamma^{s c}$ for some $\theta \in \mathscr{F}$. This gives the required result.

To answer the second question we first note that $G(\sigma)=G(\sigma)^{u} T(\sigma)$ and $T \subseteq C^{0}$. This implies that $C(\sigma) / C^{0}(\sigma) \simeq C \cap G(\sigma)^{u} / C^{0} \cap G(\sigma)^{u}$ and thus we need only describe $C^{0} \cap G(\sigma)^{u}$.

Let $X^{a d}(\mu), X^{e}(\mu), \cdots$ denote the lattices in $X$ associated with $C^{0}$. Thus $X^{a d}(\mu)$ is spanned by $\Sigma(\mu)=\{\alpha \in \Sigma: \mu(\alpha) \in d Z\}$ (this is the $\Sigma(\varphi)$ of 4.1) and $X(\mu)=X$. Let $\Delta^{s c}=\Delta^{s c}\left(C^{0}\right)$ be defined as in 5.2 (but for $C^{0}$ ) and let $\Delta, \Delta^{e}$ denote the images of $X(\mu)$ and $X^{e}(\mu)$ respectively. Both $\Delta$ and $\Delta^{e}$ depend on $X$ as it varies between $X^{a d}$ and $X^{s c}$ (the root and weight lattices of $G$ ). Let $\Delta(s c), \Delta^{e}(s c)$ denote their values when $X=X^{s c}$.

Put $L(\mu)$ and $J(\mu)$ for the subgroups associated with $C^{0}$ as in 5.2. Since $L(\mu) \subseteq G(\sigma)^{u}$ the structure of $C^{0} \cap G(\sigma)^{u}$ is determined once $J(\mu)^{u}=J(\mu) \cap G(\sigma)^{u}$ and $D(\mu)^{u}=C^{0} \cap G(\sigma)^{u} / L(\mu) J(\mu)^{u}$ are given. Now $J(\mu)^{u}=\operatorname{Ann}\left(\tau_{*} X^{s c} \cap X+X^{a d}(\mu)\right)$ and hence $J(\mu)^{u} \simeq$ 
$X /\left(\tau_{*} X^{s c} \cap X+X^{a d}(\mu)\right)$. If $C^{0}$ is semi-simple this simplifies to $J(\mu)^{u} \simeq$ $\Delta / \Delta \cap \tau_{*} \Delta(s c)$. A slight extension of the arguments in the proof of proposition $H$ gives $D(\mu)^{u} \simeq \operatorname{Ker} \tau_{*} / \Delta^{e}(s c) \cap \operatorname{Ker} \tau_{*}$.

5.5. Field automorphisms. Let $G$ be semi-simple. Suppose $\rho$ is a morphism of $G$ onto itself such that $\rho^{f}=\sigma$ for some integer $f \geqq$ 2. Then $\rho$ induces an automorphism of order $f$ on $G(\sigma)$ whose centralizer is clearly $G(\rho) \subseteq G(\sigma)$. We call the restriction of $\rho$ to $G(\sigma)$ a 'field automorphism'. If $X=X^{\text {ad }}$ the field automorphisms together with those induced by $G(\sigma)$ (inner and diagonal) and by any $\psi \neq 1$ (graph) give a complete set of representatives for Aut $(G(\sigma))$. If $X \neq X^{a d}$ the only difference is that the diagonal automorphisms occur in $N_{G}(G(\sigma))$.

An example may clarify the implications of our definition of a field automorphism. Let $G$ be of type $E_{6}\left(\right.$ and $\left.X=X^{a d}\right)$ and let $\psi$ denote its graph automorphism of order 2. Put $\sigma_{q}$ for the Frobenius morphism of $G$ whose fixed points on $k$ is the Galois field $\mathbf{F}(q)$, with $q=p^{m}$. We may suppose that $\psi$ and $\sigma_{q}$ are in standard form relative to a fixed $\Pi$ and hence $\psi \sigma_{q}=\sigma_{q} \psi$. Put ${ }^{2} \sigma_{q}=\psi \sigma_{q}$ then $G(\sigma)^{u} \simeq E_{6}(q)$ or ${ }^{2} E_{6}(q)$ depending on $\sigma=\sigma_{q}$ or ${ }^{2} \sigma_{q}$. Suppose $m$ is even, then $G\left(\sigma_{q}\right)$ has two field automorphisms of order 2, namely $\sigma_{\vee_{q}}$ and ${ }^{2} \sigma_{\vee_{q}}$; however $G\left({ }^{2} \sigma_{q}\right)$ has none. Of course $G\left(\sigma_{q}\right)$ and $G\left({ }^{2} \sigma_{q}\right)$ both have two classes of graph automorphisms of order 2 (see 4.3, the centralizers are isomorphic to $F_{4}(q)$ or $\left.C_{4}(q)\right)$.

Consider $\rho$ as restricted to $G(\sigma)$, then $\langle G(\sigma), \rho\rangle$ is a semi-direct product (with $\rho^{f}=1$ ). If $g \in G(\sigma)$ and $(g \rho)^{f}=1$ an application of Lang's theorem shows that $g \rho$ is conjugate to $\rho$ under the action of $G(\sigma)$.

In practice it is useful to know the structure of $G(\rho) \cap G(\sigma)^{u}$. If $X=X^{a d}$ one need only determine the diagonal part $D(\rho)^{u} \equiv$ $G(\rho) \cap G(\sigma)^{u} / G(\rho)^{u}$. The usual lattice calculations give $D(\rho)^{u} \simeq$ $\operatorname{Ker}\left(\rho_{*}-1\right) / \operatorname{Ker}\left(\rho_{*}-1\right) \cap \operatorname{Im}\left(1+\rho_{*}+\cdots+\rho_{*}^{f-1}\right)$, computed in $\Delta^{s c}(G)$.

5.6. Examples. We consider the $D_{4}$ example discussed in 4.5. Let $\sigma$ be in standard form relative to $\Pi$ and suppose $\sigma_{*} \alpha_{1}=q \alpha_{2}$, $\sigma_{*} \alpha_{2}=q \alpha_{1}, \sigma_{*} \alpha_{3}=q \alpha_{3}, \sigma_{*} \alpha_{4}=q \alpha_{4}$ where $q=p^{m}, p=$ characteristic of $k$. Thus $G(\sigma)^{u} \simeq{ }^{2} D_{4}(q)$. We assume $q$ is odd and consider the involution $\eta_{3}(-1) ; \quad$ since $(\sigma-1) \eta_{3}=(q-1) \eta_{3} \in 2 \Gamma$ we have $\eta_{3}(-1) \in G(\sigma)$. Since $V\left(\eta_{3}\right)=V \simeq Z_{2} \times Z_{2}, \quad \tilde{C} \simeq V /(1-\sigma) V$ giving coset representatives $1, n_{1}$, where $v_{1}=w\left(n_{1}\right)$. Hence $\left[\eta_{3}\right]$ splits into the two classes $\left[\eta_{3}, \sigma\right]$ and $\left[\eta_{3}, \sigma_{1}\right]$ where $\sigma_{1}=i_{n_{1}} \sigma$.

Since $\frac{1}{2}(\sigma-1) \eta_{3} \in \Gamma^{s c}$ prop. $\mathrm{H}$ gives $\eta_{3}(-1) \in G(\sigma)^{u}$, however $\frac{1}{2}\left(\sigma_{1}-1\right) \eta_{3}=-q \eta_{1}+((q-1) / 2) \eta_{3} \equiv \eta_{1}\left(\bmod \Gamma^{s c}\right) \quad$ and, since $\eta_{1} \notin$ 
$\left(\sigma_{1}-1\right) \Gamma, \eta_{3}(-1) \notin G\left(\sigma_{1}\right)^{u}$. Thus the class $\left[\eta_{3}, \sigma\right]$ is "inner" (in fact central) while $\left[\eta_{3}, \sigma_{1}\right]$ is outer-diagonal.

We now describe the structure of $C(\sigma)$ and $C\left(\sigma_{1}\right)$ where $C$ is given in 4.5. We use the methods of 5.2. From the permutations induced on $\left\{\alpha_{1}, \alpha_{2}, \alpha_{4}, \alpha_{*}\right\}$ by $\sigma$ and $\sigma_{1}$ we have $L / Z \simeq A_{1}\left(q^{2}\right) \times A_{1}(q) \times A_{1}(q)$ for $\sigma$ and $L / Z \simeq A_{1}\left(q^{4}\right)$ for $\sigma_{1}$. Next let $\Delta^{s c}=\left\langle\epsilon_{1}, \epsilon_{2}, \epsilon_{4}, \epsilon_{*}\right\rangle \simeq$ $Z_{2} \times Z_{2} \times Z_{2} \times Z_{2}$ where $\epsilon_{i}$ is the image of the fundamental weight $\alpha_{\imath} / 2$. Thus $\Delta=\Delta^{e}=\left\langle\epsilon_{1}+\epsilon_{2}+\epsilon_{4}+\epsilon_{*}\right\rangle$ and (see 5.4) $\Delta(s c)=\Delta^{e}(s c)=$ $\left\langle\epsilon_{1}+\epsilon_{2}, \epsilon_{2}+\epsilon_{4}, \epsilon_{4}+\epsilon_{*}\right\rangle$. We now calculate in $\Delta^{s c}$;

\begin{tabular}{|c|c|c|c|c|}
\hline & $\operatorname{Ker} \tau_{*}$ & $\operatorname{Im} \tau_{*}$ & $\tau_{*} \Delta$ & $\Delta \cap \tau_{*} \Delta(s c)$ \\
\hline$\sigma$ & $\left\langle\epsilon_{1}+\epsilon_{2}, \epsilon_{4}+\epsilon_{*}\right\rangle$ & $\left\langle\epsilon_{1}+\epsilon_{2}\right\rangle$ & 0 & 0 \\
\hline$\sigma_{1}$ & $\Delta$ & $\Delta(s c)$ & 0 & $\Delta$ \\
\hline
\end{tabular}

Using proposition $\mathrm{G}$ and 5.4 we thus have:

\begin{tabular}{|cccc|cc|}
\hline & $Z$ & $J$ & $D$ & $J^{u}$ & $D^{u}$ \\
\hline$\sigma$ & $Z_{2}$ & $Z_{2}$ & $Z_{2} \times Z_{2}$ & $Z_{2}$ & $Z_{2}$ \\
\hline$\sigma_{1}$ & 1 & $Z_{2}$ & 1 & 1 & 1 \\
\hline
\end{tabular}

So, for example, $C^{0}\left(\sigma_{1}\right) \simeq A_{1}\left(q^{4}\right) \times Z_{2}$ while $C^{0} \cap G\left(\sigma_{1}\right)^{u} \simeq A_{1}\left(q^{4}\right)$.

The only coset of $C / C^{0}$ fixed by $\sigma$ (or $\sigma_{1}$ ) is $n_{4} C^{0}$. From the standard form of $n_{4}$ we have $\left[n_{4}, \sigma\right]=\left[n_{4}, \sigma_{1}\right]=1$ and so the structure of $C(\sigma), \quad C\left(\sigma_{1}\right)$ is easily computed. For example, put $x_{+}(\zeta)=$ $x_{\alpha_{1}}(\zeta) x_{\alpha_{2}}\left(\zeta^{q^{3}}\right) x_{\alpha_{4}}\left(\zeta^{q^{2}}\right) x_{\alpha_{0}}\left(\zeta^{q^{4}}\right)$ and define $x_{-}$similarly, then $C^{0}\left(\sigma_{1}\right)=$ $\left\langle x_{ \pm}(\zeta): \zeta \in \mathbf{F}\left(q^{4}\right)\right\rangle \times\left\langle\eta_{3}(-1)\right\rangle$. Since $n_{4} x_{ \pm}(\zeta) n_{4}^{-1}=x_{ \pm}\left(\zeta^{q^{2}}\right) \quad$ we have $C\left(\sigma_{1}\right) \simeq \mathrm{FL}_{2}\left(q^{4}\right) \times Z_{2}$ (see the first paragraph of Appendix 1 for notation).

Appendix 1. We list the pairs $(L, t)$ where $L$ is a finite simple Chevalley type group over a field of odd characteristic and $t$ is an involution in Aut $L$ such that the layer of $C_{L}(t)$ consists of a single $\mathrm{PSL}_{2}$-group. We also describe $C_{L}(t)$. The classes are those occurring under conjugation by $G(\sigma)$ where $L=G(\sigma)^{u}$. The results are given in the table below. The notation is as follows: $q=p^{m}, p$ some odd prime; $L_{2}(q)=\operatorname{PSL}_{2}(q)$ 
$\mathrm{PGL}_{2}(q)$ is $L_{2}(q)$ extended by diagonal automorphism $\mathrm{FL}_{2}\left(q^{2}\right)$ is $L_{2}\left(q^{2}\right)$ extended by field aut. (of order 2)

$\operatorname{PFL}_{2}\left(q^{2}\right)$ is $L_{2}\left(q^{2}\right)$ extended by (field) $\times$ (diagonal) aut. For example, if $q=3, \mathrm{FL}_{2}(9) \simeq \Sigma_{6}$ and $\mathrm{PFL}_{2}(9) \simeq M_{10} . \quad Z_{n}$ and $D_{n}$ are respectively the cyclic and dihedral group of order $n$. In the entry for ${ }^{2} \mathrm{D}_{4}(3) \mathrm{H}$ is a four-dimensional orthogonal group; it has a subgroup $\mathrm{H}_{0}$ of index 2 with $H_{0} \simeq \mathrm{SL}_{2}(3) * \mathrm{SL}_{2}(3)$ (a central product) and $s$ flips these factors.

We sketch the calculations: Let $G$ be a simple algebraic group with $X=X^{a d}$ and $\sigma$ a morphism so that $L=G(\sigma)^{u}$. We consider the various involutions $t$ in Aut $L$; (i) $t$ a field automorphism, then $G$ is either of type $A_{1}$ or we have the exceptional case $G_{2}$ as given in the table,

L $\quad t$

$C_{\mathrm{L}}(t)$

\begin{tabular}{|c|c|c|c|}
\hline$A_{1}\left(q^{2}\right)$ & field & $q \neq 3$ & $\mathrm{PGL}_{2}(q)$ \\
\hline${ }^{2} G_{2}(q)$ & inner & $q=3^{2 m+1}, \quad m \geqq 1$ & $L_{2}(q) \times Z_{2}$ \\
\hline$A_{2}(q)$ & graph & $q \neq 3$ & $\mathrm{PGL}_{2}(q)$ \\
\hline${ }^{2} A_{2}(q)$ & graph & $q \neq 3$ & $\mathrm{PGL}_{2}(q)$ \\
\hline$C_{2}(q)$ & $\begin{array}{l}\text { inner } \\
\text { diagonal } \\
\text { diagonal }\end{array}$ & $q \equiv \epsilon(4), q \neq 3$ & $\left.\begin{array}{c}\left\langle L_{2}(q) \times D_{q-\epsilon}, \tau\right\rangle \\
\left\langle L_{2}(q) \times D_{q+\epsilon}, \tau\right\rangle\end{array}\right\} \begin{array}{l}\tau^{2}=1,\left\langle L_{2}(q), \tau\right) \simeq \operatorname{PGL}_{2}(q) \\
\left\langle D_{q \mp \epsilon}, \tau\right\rangle \simeq D_{2(q \mp \epsilon)}\end{array}$ \\
\hline${ }^{2} D_{4}(q)$ & diagonal & & $\mathrm{FL}_{2}\left(q^{4}\right)$ \\
\hline$A_{3}(q)$ & $\begin{array}{l}\text { diagonal } \\
\text { inner } \\
\text { graph }\end{array}$ & $\begin{array}{l}q \equiv 1(4) \\
q \equiv-1(4) \\
\left\{\begin{array}{l}q \equiv 1(8) \\
q \equiv 3,7(8) \\
q \equiv 5(8)\end{array}\right.\end{array}$ & $\left.\begin{array}{c}\left\langle L_{2}\left(q^{2}\right) \times Z_{(q+1) / 2}, \theta\right\rangle \\
\left\langle L_{2}\left(q^{2}\right) \times Z_{(q+1)}, \theta\right\rangle\end{array}\right\} \begin{array}{l}\theta^{2}=1,\left\langle L_{2}\left(q^{2}\right), \theta\right\rangle \approx \mathrm{FL}_{2}\left(q^{2}\right) \\
\mathrm{FL}_{2}\left(q^{2}\right) \\
\operatorname{PGL}_{2}\left(q^{2}\right) \\
\operatorname{PFL}_{2}\left(q^{2}\right)\end{array}$ \\
\hline${ }^{2} A_{3}(q)$ & $\begin{array}{l}\text { inner } \\
\text { diagonal } \\
\text { graph }\end{array}$ & $\begin{array}{l}q \equiv 1(4) \\
q \equiv-1(4) \\
\left\{\begin{array}{l}q \equiv 1,5(8) \\
q \equiv 3(8) \\
q \equiv 7(8)\end{array}\right.\end{array}$ & $\begin{array}{r}\left.\begin{array}{l}\left\langle L_{2}\left(q^{2}\right) \times Z_{q-1}, \theta\right\rangle \\
\left\langle L_{2}\left(q^{2}\right) \times Z_{(q-1) / 2}, \theta\right\rangle\end{array}\right\} \theta \text { as for } A_{3}(q) \\
\operatorname{PGL}_{2}\left(q^{2}\right) \\
\operatorname{PFL}_{2}\left(q^{2}\right) \\
\operatorname{FL}_{2}\left(q^{2}\right)\end{array}$ \\
\hline $\begin{array}{l}G_{2}(3) \\
B_{3}(3) \\
{ }^{2} D_{4}(3)\end{array}$ & $\begin{array}{l}\text { graph } \\
\text { diagonal } \\
\text { inner }\end{array}$ & & $\begin{array}{l}{ }^{2} G_{2}(3) \approx\left\langle L_{2}\left(2^{3}\right), f\right\rangle, f^{3}=1, \text { field aut. } \\
\quad \operatorname{PGL}_{2}(3) \times \mathrm{FL}_{2}(q) \\
\left\langle H \times L_{2}(q), s\right\rangle s^{2}=1,\left\langle L_{2}(9), s\right\rangle=\mathrm{FL}_{2}(9) \\
\text { (see text for } H \text { and action of } s \text { ) }\end{array}$ \\
\hline
\end{tabular}


(ii) $t$ is a graph automorphism, then Table 4.3 shows that $G$ is of type $A_{2}$ or $A_{3}$, (iii) $t$ is inner (in $G$ ), i.e., the case $\psi=1$ in $\$ 3,4$, then it is easily seen that $G$ has rank $\leqq 4$. At this stage it is necessary to list the various cases, compute $C^{0}=C_{G}(t)^{0}$ and $O^{P^{\prime}}\left(C^{0}(\sigma)\right)$, and see when the layer is an $L_{2}$-group. The calculations given in 5.6 are typical.

The authors wish to kindly thank M. Harris and L. Finkelstein for pointing out errors in the original version of this table.

APPENDIX 2. We use the notation of $\S 2$, especially 2.4 . For a given $\mu \in \Gamma$ we describe an algorithm which finds the unique $\lambda \in \mathscr{F}_{d}$ such that $\mu$ and $\lambda$ lie in the same $\mathscr{A}_{d}^{s c}$-orbit in $\Gamma$. This algorithm is an essential tool in practical calculations (especially if $d>3$ ).

Without restriction we may suppose that the Dynkin diagram for $\Sigma$ is connected. Let $\Pi=\left\{\alpha_{1}, \cdots, \alpha_{r}\right\}$ and let $\left\{\eta_{1}, \cdots, \eta_{r}\right\}$ be the dual basis for $\Gamma^{a d}$, i.e. $\eta_{t}\left(\alpha_{l}\right)=\delta_{i v}$. There is a natural inclusion $\Gamma \subseteq \Gamma^{a d}$ and if $\Gamma \neq \Gamma^{a d}$ an element $\eta=\Sigma e_{i} \eta_{t}\left(e_{t} \in Z\right)$ lies in $\Gamma$ if and only if certain (easily found) congruences hold. Let $\alpha_{*}=-\left(m_{1} \alpha_{1}+\cdots+m_{r} \alpha_{r}\right)$ where the $m_{\imath}$ are (known) positive integers then $\mathscr{F}_{d}(\Gamma)=\left\{\eta \in \Gamma: 0 \leqq e_{t}\right.$ all $i$ and $\left.\left(m_{1} e_{1}+\cdots+m_{r} e_{r}\right) \leqq d\right\}$.

Now suppose $\mu=\Sigma a_{\imath} \eta_{t} \in \Gamma\left(a_{\imath} \in Z\right)$ the algorithm goes as follows (let $w_{i}=w_{\alpha_{i}}$ and $w_{*}=w_{\alpha_{.}}$);

(1) if some $a_{\imath}<0$ replace $\mu$ by $w_{i} \mu$,

(2) if all $a_{\imath} \geqq 0$ but $m_{1} a_{1}+\cdots+m_{r} a_{r}>d$ replace $\mu$ by $w_{*} \mu-d \hat{\alpha}_{*}$.

By repeating these two steps we eventually obtain an element of $\mathscr{F}_{d}$. Note that (i) $w_{\imath} \eta_{\jmath}=\eta_{\text {, }}$ if $i \neq j$ while $w_{\imath} \eta_{i}=\eta_{i}-\hat{\alpha}_{i}$, (ii) $w_{*} \mu=$ $\mu+\left(m_{1} a_{1}+\cdots+m_{r} a_{r}\right) \hat{\alpha}_{*}$ and (iii) the expressions for $\hat{\alpha}_{i}, \hat{\alpha}_{*}$ in terms of the $\eta_{\text {, }}$ 's can be read off from the extended Dynkin diagram.

The operation in steps (1), (2) are just reflections on the bounding hyperplanes of $\mathscr{F}_{d}$. Using result 1.16 of [14] it may be shown that we have the best possible algorithm.

\section{REFERENCES}

1. E. Abe, On the groups of C. Chevalley, J. Math. Soc. Japan, 11, (1959), 15-41.

2. N. Bourbaki, Éléments de mathématique: Groupes et algèbres de Lie, Chapt. IV, V, VI. Hermann, Paris (1968).

3. N. Burgoyne, Finite groups with Chevalley type components, Pacific J. Math., 72 (1977), 341-350.

4. N. Burgoyne and C. Williamson, Centralizers of involutions in Chevalley groups, (dittoed notes).

5. N. Burgoyne, Elements of order 3 in Chevalley groups of characteristic 2., (dittoed notes).

6. Séminaire C. Chevalley, Classification des groupes de Lie algébriques, Paris, (1958).

7. R. Foote, Finite groups with maximal 2-components of type $L_{2}(q), q$ odd, (to appear).

8. D. Gorenstein, Finite Groups, Harper \& Row, N. Y., (1968).

9. M. Harris and R. Solomon, Finite groups having an involution centralizer with a 2-component of dihedral types, (to appear).

10. N. Iwahori, Centralizers of involutions in finite Chevalley groups, Lecture Notes in Math. No.

131, Springer, Berlin, (1970). 
11. S. Lang, Algebraic groups over finite fields, Amer. J. Math., 78, 555-563 (1956).

12. R. Ree, Classification of involutions and centralizers of involutions in certain simple groups, Proc.

Int. Conf. Theory of Groups, Canberra 1965, 281-301, Gordon \& Breach, N. Y. 1967.

13. T. A. Springer and R. Steinberg, Conjugacy classes, Lecture Notes in Math. No. 131, Springer, Berlin (1970).

14. R. Steinberg, Endomorphisms of linear algebraic groups, Mem. Amer. Math. Soc. No. 80 (1968).

Received July 1, 1976 and in revised form January 26, 1977. The work by the first author was supported by NSF Grant MPS74-07807.

UNIVERSITY OF CALIFORNIA

SANTA CRUZ, CA 95064 



\section{Pacific Journal of Mathematics \\ Vol. 70, No. $1 \quad$ September, 1977}

William H. Barker, Noether's theorem for plane domains with hyperelliptic

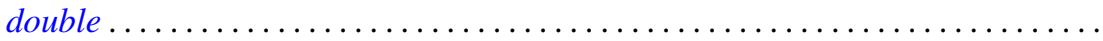

Michael James Beeson, Non-continuous dependence of surfaces of least area on the boundary curve ...................................... 11

Horst Behncke, Functions acting in weighted Orlicz algebras . . . . . . . . . . . . 19

Howard Edwin Bell, A commutativity study for periodic rings . . . . . . . . . . . 29

Peter Botta and Stephen J. Pierce, The preservers of any orthogonal group ....... 37

Douglas S. Bridges, The constructive Radon-Nikodým theorem ............. 51

James Dennis Brom, The theory of almost periodic functions in constructive

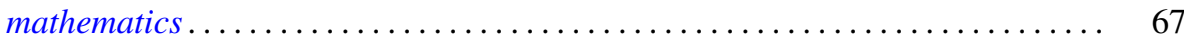

N. Burgoyne and C. Williamson, Semi-simple classes in Chevalley type groups ....

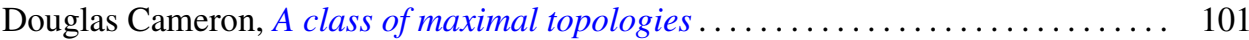

L. Carlitz, Enumeration of doubly up-down permutations . . . . . . . . . . . . . . 105

Paul Robert Chernoff, The quantum n-body problem and a theorem of

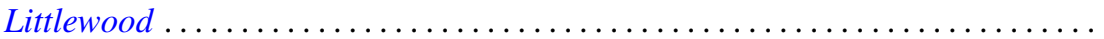

Jo-Ann Deborah Cohen, Locally bounded topologies on $F(X) \ldots \ldots \ldots \ldots \ldots \ldots$

Heinz Otto Cordes and Robert Colman McOwen, Remarks on singular elliptic

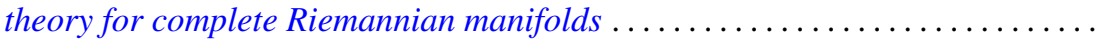

Micheal Neal Dyer, Correction to: "Rational homology and Whitehead

products"

Robert Fernholz, Factorization of Radonifying transformations

Lawrence Arthur Fialkow, A note on quasisimilarity. II ...... . .

Harvey Charles Greenwald, Lipschitz spaces of distributions on the surface of unit



Albrecht Irle, On the measurability of conditional expectations

Tom (Roy Thomas Jr.) Jacob, Matrix transformations involving simple sequence



A. Katsaras, Continuous linear maps positive on increasing continuous

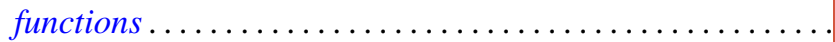

Kenneth Kunen and Judith Roitman, Attaining the spread at cardinals of cofinality

Lawrence Louis Larmore and Robert David Rigdon, Enumerating normal bundles

of immersions and embeddings of projective spaces ...... . .

Ch. G. Philos and V. A. Staïkos, Asymptotic properties of nonoscillatory solutions of differential equations with deviating argument .

Peter Michael Rosenthal and Ahmed Ramzy Sourour, On operator algebras containing cyclic Boolean algebras...

Polychronis Strantzalos, Strikt fast gleichgradig-stetige und eigentliche

Aktionen ...

Glenn Francis Webb, Exponential representation of solutions to an abstract

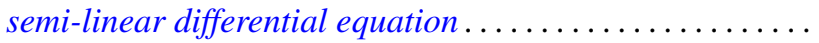

